\title{
Article \\ The Use of Traditional Korean Medicine (TKM) by Children: A Correlational Study between Parent's Perception and Their Children's Use Reported by Parents
}

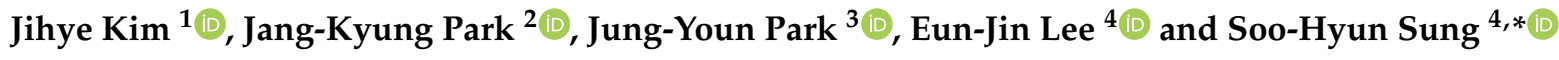 \\ 1 Research Institute of Korean Medicine Policy, The Association of Korean Medicine, Seoul 07525, Korea; \\ jihyekim1217@gmail.com \\ 2 Department of Obstetrics and Gynecology, College of Korean Medicine, Pusan National University, \\ Yangsan 50612, Korea; vivat314@pusan.ac.kr \\ 3 Department of Health and Welfare, Yuhan University, Bucheon 14780, Korea; park0625@yuhan.ac.kr \\ 4 Department of Policy Development, National Development Institute of Korean Medicine, Seoul 04554, Korea; \\ eunjin6434@nikom.or.kr \\ * Correspondence: koyote10010@nikom.or.kr
}

\section{check for}

updates

Citation: Kim, J.; Park, J.-K.; Park, J.-Y.; Lee, E.-J.; Sung, S.-H. The Use of Traditional Korean Medicine (TKM) by Children: A Correlational Study between Parent's Perception and Their Children's Use Reported by Parents. Healthcare 2021, 9, 385. https://doi.org/10.3390/ healthcare 9040385

Academic Editors: Manoj Sharma and Kavita Batra

Received: 17 February 2021

Accepted: 26 March 2021

Published: 1 April 2021

Publisher's Note: MDPI stays neutral with regard to jurisdictional claims in published maps and institutional affiliations.

Copyright: (c) 2021 by the authors. Licensee MDPI, Basel, Switzerland. This article is an open access article distributed under the terms and conditions of the Creative Commons Attribution (CC BY) license (https:// creativecommons.org/licenses/by/ $4.0 /)$.

\begin{abstract}
This cross-sectional study investigated the correlation between parents' perception and their children's traditional Korean medicine (TKM) use reported by parents in order to discover policy intervention points and provide a reference for establishing generalized TKM policies. Participant data from a 2017 national survey on TKM usage was divided into two groups based on the children's TKM use reported by parents. The female participants' children had a higher rate of experience in using TKM $(8.1 \% ; p=0.029)$. Additionally, $91.4 \%$ of the parent group with a child who used TKM turned out to have used TKM, which was higher than $71.9 \%$ of the parents whose children never used TKM $(p<0.001)$. As for the awareness on the use of TKM, $44.0 \%$ of the parents with a child who experienced TKM answered they were aware of it, while only $35.3 \%$ of the parent group whose child never experienced TKM did so $(p=0.033)$. The present study suggests that parental experience in using TKM could have an impact on the children's TKM use reported by parents. Further study is necessary to assess which parental factor (awareness level, medical disorder to be treated, therapy, therapeutic efficacy, the purpose of visit, sex, age, etc.) has a close relationship with TKM usage experience of their children.
\end{abstract}

Keywords: traditional Korean medicine (TKM); complementary and alternative medicine (CAM); national survey TKM usage; parents' perception of TKM; children's TKM use

\section{Introduction}

Complementary and Alternative Medicine (CAM), which is not considered to be part of conventional medicine, is a compilation of knowledge, skills, and practices which are based on the theories, beliefs, and experiences indigenous to different cultures and used for health maintenance and in the prevention, diagnosis, improvement, or treatment of physical and mental illness [1-3]. CAM approaches include natural products (e.g., herbs, vitamins and minerals and probiotics), and mind body practice such as yoga, meditation, chiropractic, acupuncture, relaxation techniques, tai chi, qigong, and hypnotherapy [4]. The use of CAM is increasing in many countries of the world [5]. Approximately $88 \%$ of the member states of the World Health Organization (WHO) are using CAM through the development of national policies, laws, regulations, and applied programs [6]. In EastAsian countries such as South Korea and China, traditional medicine has been the form of medical care treating the diseases of the people. Currently, it is still taking a crucial part in health care along with conventional medicine (CM) $[7,8]$. In East-Asian countries such as China, Korea, and Taiwan, traditional medicine practitioners are considered as doctors, as is the case with the doctors who provide CM $[9,10]$. Traditional Korean medicine (TKM) 
doctors use acupuncture, electro-acupuncture, pharmacopuncture, herbal medicine, chuna, cupping, moxibustion, and other forms of intervention to treat their patients [11]. In Korea, $14 \%$ of the total population and $7.6 \%$ of those in their $20 \mathrm{~s}$ or younger, are using TKM, and $10 \%$ of the total male population and $18.1 \%$ of the total female population visited TKM clinics [12]. The purpose of using TKM was treating a disease (94.1\%), improving health $(18.4 \%)$, and cosmetic purposes $(4.0 \%)$ [13].

Health in the pediatric or juvenile period has an impact on adulthood health, education, achievement, and economic performance. Therefore, the health of children and teenagers is of paramount importance [14,15]. Additionally, parents' experience with using medical services can have varying effects on their children [16]. Some well-known factors impacting the health and the usage of medical services during pediatric and juvenile periods include respective family structure, parental education level, and their social- status [17-22].

In the field of traditional Chinese medicine (TCM), Loh [23] surveyed 300 parents who visited TCM clinics, and $84.3 \%$ of their children used TCM clinics, while $80.3 \%$ of them reported that they used both TCM clinics and CM clinics. Yeh [24] used the National Health Insurance data of Taiwan to analyze the overall usage of TCM and reported that about $20 \%$ of the children under 20 used TCM clinics. In the field of TKM, Choi [25] conducted a survey of 300 parents who used the TKM clinic and had a child under 19. As a result of the said survey, it was resulted that $81 \%$ of the children experienced and visited TKM clinics for the purpose of treating respiratory disease $(21.6 \%)$ gastrointestinal disease $(10.6 \%$,), and skin disease (9.2\%). Park [26] surveyed 702 parents who used daycare centres and reported that $55.3 \%$ of the children's age from 1 to 13 used TKM clinics, mainly for the purpose of treating respiratory disease $(34.5 \%)$, gastrointestinal disease $(17.2 \%)$, and skin disease $(13.8 \%)$. As such, the previous studies on the usage of traditional medicine among children or adolescents under 20, were mainly conducted as cross-sectional studies. In three studies $[23,25,26]$, the study samples were not representative of the general population of the country. Yeh [24] used the representative data of the general population of Taiwan. However, the study was intended to investigate the usage of TCM in the entire population, including children.

An assessment of TKM usage has been undertaken by Statistics Korea, as a certified national statistic every 3 years since 2008. This assessment covers all household members of the sample families who are at least 19 years old, providing a representative sample of the country [13]. In order to effectively integrate TKM into the healthcare system, it is necessary that policies are developed and implemented based on accurate statistics of TKM. To fulfil this requirement, the national survey on TKM use data from 5000 Korean participants was used in order to examine the correlation between parental awareness of TKM use and the use of TKM by their children.

\section{Materials and Methods}

\subsection{Data Sources}

The source data used in this study is from the TKM usage survey of 2017, conducted by the Ministry of Health and Welfare and National Institute of Korean Medicine Development. The survey was reviewed and approved by Korea Statistics (National Certified Statistics Approval Number: 117087). As for the survey data, the researchers made a request for micro-data through the surveys on the traditional herbal medicines consumption and the TKM usage database (https: / / www.koms.or.kr/main.do, accessed on 18 November 2020) run by the National Institute of Korean Medicine Development (NIKOM). Approval was obtained to use and analyze the data from the NIKOM, which was commissioned by the government to conduct the above-mentioned survey and analyzed the micro-data which was provided by the said organization to conduct this study.

As for the surveys on the traditional herbal medicine consumption and the TKM usage, the survey on the usage of TKM started in 2008 (1st survey), which was followed by the survey on the consumption of traditional herbal medicine (1st survey) in 2009. In 2011, 
the two separate surveys were integrated into one survey on TKM usage. Since then, the survey has been conducted every 3 years. Among all the data collected over the years, we used the data on the usage of TKM obtained during the 4th survey that took place in 2017.

\subsection{Sample Selection}

The data used in this study was obtained from 5000 participants selected among the general population during the TKM usage survey in 2017. When the 5000 participants, who were the national survey samples, were selected, they did not enroll both parents from a family. In particular the following selecting questions were used to assess eligibility: Do you have a child who is under 19 (born after September 1998)? (1) Yes [the number of children under 19 is (...)] (2) No. The participants in the survey item or were the parent who was the caregiver of the children. Also, we instructed the participants to tell us the number of children in their family if they answered they had a child under 19 . This resulted in the inclusion of 872 participants who chose 'Yes'. Their answers were included for analysis in order to obtain the results on the satisfaction and awareness data, as well as the information regarding their children. The selection process of the participants is shown in Figure 1.

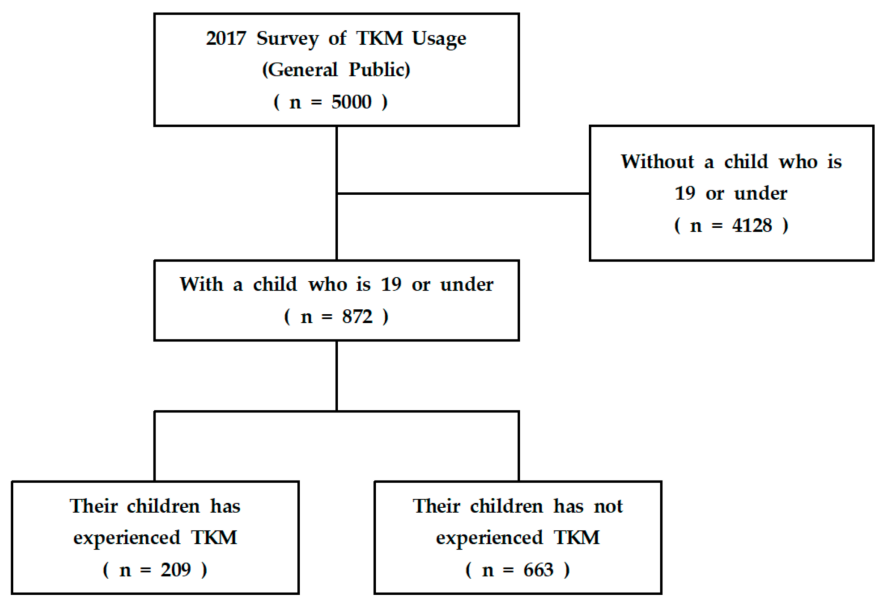

Figure 1. Flow chart of inclusion and exclusion of participants from the 2017 National Survey of Traditional Korean Medicine Usage. TKM: Traditional Korean Medicine.

\subsection{Analysis Items}

The analysis items included in this study were the participants who answered 'Yes' to the question asking whether they had a child under the age of 19, during the TKM usage survey in 2017, as well as the questions regarding the satisfaction and awareness of their children. Additionally, based on the children's TKM use reported by parents, the relationships between the demographics, the experience of using TKM, their opinion on the use of TKM, and the perception of treatment effects on sixteen diseases, were analyzed. The demographics of the participants (mother or father) were used to examine the relationship between the answers about the children and the characteristics of participants. The answers about the children included the children's experience with TKM, the reason why their children used TKM, the kind of TKM treatment the children experienced, the degree of satisfaction with their children's TKM experience, and cause of dissatisfaction with using TKM. The basic demographic information of the participants included their sex, age group, area of residence, education, employment status, household income, type of healthcare coverage, and subscription status of private medical insurance. The experience of using TKM variables included TKM participation, the reason for choosing to use TKM, participants' experience with TKM, participants' satisfaction with TKM, participants' awareness of TKM, money invested to use TKM, their willingness to use TKM again in the future, and their willingness to recommend TKM to others. Regarding the perception of treatment effects on diseases, variables were used following sixteen diseases: (1) disc related disease (herniation of intervertebral disc, spinal stenosis); (2) osteoarthritis; (3) frozen shoulder shoulder pain; 
(4) back pain; (5) sprain; (6) facial nerve paralysis; (7) stroke; (8) hypertension; (9) diabetes mellitus; (10) digestive disease; (11) common cold rhinitis; (12) dementia (13) cancer related pain; (14) infertility; (15) skin disease (atopic dermatitis); (16) genitourinary disease. Also, whether the characteristics of the participants (demographics, their opinion or ideas on TKM, etc.) had an impact on the children's use of TKM and the possible strategies to promote use of TKM in different target groups, were explored.

\subsection{Statistical Analysis}

In order to better understand the general characteristics of the participants included for analysis, the frequencies and ratios were calculated. Based on the children's TKM use reported by parents, the correlations between variables were analyzed using the chi-square test $\left(\chi^{2}\right.$-test), which is a cross-analysis method of the ratios between different groups and is commonly used when analyzing categorized data. IBM SPSS Statistics for Windows, version 25 (IBM Corp., Armonk, NY, USA) was used for all statistical analyses and the significance level was set at $5 \%(p<0.05)$.

\section{Results}

\subsection{The Information on and Characteristics of the Children of the Participants}

The basic statistics of the 5000 participants (fathers or mothers) from the general population who participated in the survey were obtained based on their answers to the question regarding their children (Table 1). Regarding the question about their children, 872 participants answered that they had a child (or children) under the age of 19, accounting for $17.4 \%$ of all participants (Table 1 ). The majority of these contributors answered that they only had one child that fell into that particular age group ( $n=425,48.7 \%)$, which was followed by those with two children in the corresponding age group $(n=393,45.1 \%)$. When asked whether their children had any experience of using TKM over the past 12-month period, 209 answered they had, while 663 answered none (24.0\% and $76.0 \%$, respectively).

Table 1. Information and characteristics of the respondent's children.

\begin{tabular}{llll}
\hline Category & & & $n(\%)$ \\
\hline \multirow{2}{*}{ Existence of chlidren under 19 } & Yes & No & $872(17.4)$ \\
& Yes & $4128(85.6)$ \\
\hline TKM experience of children & No & & $209(24.0)$ \\
reported by parents & Number of children & $663(76.0)$ \\
\hline & TKM experience of children & Yes & $425(48.7)$ \\
& reported by parents & No & $78(18.4)$ \\
& Number of children & 2 & $346(81.6)$ \\
& TKM experience of children & Yes & $393(45.1)$ \\
Number and TKM experiences & reported by parents & No & $108(27.5)$ \\
of children & Number of children & 3 & $285(72.5)$ \\
& TKM experience of children & Yes & $52(6.0)$ \\
& reported by parents & No & $22(42.3)$ \\
& Number of children & 4 & $30(57.7)$ \\
& TKM experience of children & Yes & $2(0.2)$ \\
& reported by parents & No & $1(50.0)$
\end{tabular}

TKM: Traditional Korean Medicine.

\subsection{The Demographics of the Parents and Their Children's TKM Use Reported by Parents}

Differences in the children's demographics were assessed based on their (or their parents) experience with TKM (Table 2). Interestingly, female participants were more likely to have children with experience of using TKM $(p=0.029)$. Furthermore, the chance of having a child with experience of using TKM was higher in Chungcheong $(n=116,13.3 \%)$ and Gyeongsang $(n=225,25.8 \%)$ areas compared to the capital area $(n=371,42.5 \%)$. Participants in their $50 \mathrm{~s}$ (50 to 60 years or older) showed a higher value compared to 
participants that were 40 years old or younger (30 to 40 years or younger) $(p=0.042)$. There was also no difference in employment status in terms of the children's TKM usage reported by parents. No tendency of significance was observed with the household income as well. Participants with higher education levels (university or higher education) were more likely to answer that their children had experienced using TKM. However, the difference was not statistically significant. As for the types of government health care coverage, the groups without a child who experienced using TKM had a higher rate of having workplace health insurance coverage. Similarly, the group with children with experience of using TKM had a higher rate of having private medical insurance. However, these differences were non-statistically significant. Additionally, no significant differences were observed based on household income or area of residence.

Table 2. Characteristics of the study population from the survey with and without traditional Korean medicine (TKM) experience of children.

\begin{tabular}{|c|c|c|c|c|c|}
\hline & \multirow{2}{*}{ Category } & \multicolumn{2}{|c|}{$\begin{array}{c}\text { Existence of TKM Experience of } \\
\text { Children }\end{array}$} & \multirow{2}{*}{$\begin{array}{l}\text { Total } \\
n(\%)\end{array}$} & \multirow{2}{*}{$\chi^{2}(p)$} \\
\hline & & $\begin{array}{c}\text { Yes } \\
n(\%)\end{array}$ & $\begin{array}{c}\text { No } \\
n(\%)\end{array}$ & & \\
\hline \multirow[b]{2}{*}{ Parent Gender } & Male & $57(27.3)$ & $235(35.4)$ & $292(33.5)$ & \multirow{2}{*}{$\begin{array}{c}4.765 \\
(p=0.029)\end{array}$} \\
\hline & Female & $152(72.7)$ & $428(64.6)$ & $580(66.5)$ & \\
\hline \multirow{4}{*}{ Residence } & Metropolitan & $78(37.3)$ & $293(44.2)$ & $371(42.5)$ & \multirow{4}{*}{$\begin{array}{c}3.793 \\
(p=0.285)\end{array}$} \\
\hline & Chungcheng province & $31(14.8)$ & $85(12.8)$ & $116(13.3)$ & \\
\hline & Gyeongsang province & $55(26.3)$ & $170(25.6)$ & $225(25.8)$ & \\
\hline & Jeolla province & $45(21.5)$ & $115(17.3)$ & $160(18.3)$ & \\
\hline \multirow{4}{*}{ Age } & $30 \mathrm{~s} \leq$ & $2(1.0)$ & $31(4.7)$ & $33(3.8)$ & \multirow{4}{*}{$\begin{array}{c}8.196 \\
(p=0.042)\end{array}$} \\
\hline & $40 \mathrm{~s}$ & $79(37.8)$ & $277(41.8)$ & $356(40.8)$ & \\
\hline & $50 \mathrm{~s}$ & $111(53.1)$ & $311(46.9)$ & $422(48.4)$ & \\
\hline & $\geq 60 \mathrm{~s}$ & $17(8.1)$ & $44(6.6)$ & $61(7.0)$ & \\
\hline \multirow{2}{*}{ Job } & Yes & $139(66.5)$ & 449 (67.7) & $588(67.4)$ & \multirow{2}{*}{$\begin{array}{c}0.107 \\
(p=0.744)\end{array}$} \\
\hline & No & $70(33.5)$ & $214(32.3)$ & $284(32.6)$ & \\
\hline \multirow{5}{*}{$\begin{array}{l}\text { Household } \\
\text { income }\end{array}$} & Less than 1500 USD & $20(0.5)$ & $7(1.1)$ & $8(0.9)$ & \multirow{5}{*}{$\begin{array}{c}5.036 \\
(p=0.284)\end{array}$} \\
\hline & 1500 USD less than 3000 USD & $26(12.4)$ & $105(15.8)$ & $131(15.0)$ & \\
\hline & 3000 USD less than 4500 USD & $79(37.8)$ & $267(40.3)$ & $346(39.7)$ & \\
\hline & 4500 USD less than 6000 USD & $73(34.9)$ & $218(32.9)$ & $291(33.4)$ & \\
\hline & No less than 6000 USD & $30(14.4)$ & $66(10.0)$ & $969(11.0)$ & \\
\hline \multirow{4}{*}{$\begin{array}{l}\text { Academic } \\
\text { background }\end{array}$} & Primary or lower school graduate & $1(0.5)$ & $4(0.6)$ & $5(0.6)$ & \multirow{4}{*}{$\begin{array}{c}3.034 \\
(p=0.386)\end{array}$} \\
\hline & Middle school graduate & $0(0.0)$ & $8(1.2)$ & $8(0.98)$ & \\
\hline & High school graduate & $62(29.7)$ & $210(31.7)$ & $272(31.2)$ & \\
\hline & University or higher school graduate & $146(69.9)$ & $441(66.5)$ & $587(67.3)$ & \\
\hline \multirow{3}{*}{$\begin{array}{l}\text { Medical security } \\
\text { type }\end{array}$} & $\begin{array}{l}\text { Health insurance } \\
\text { (district insurance) }\end{array}$ & $63(37.3)$ & $149(22.5)$ & $212(24.3)$ & \multirow{3}{*}{$\begin{array}{c}5.619 \\
(p=0.060)\end{array}$} \\
\hline & Health insurance & $146(69.9)$ & $512(77.2)$ & $658(75.5)$ & \\
\hline & Medical care & $0(0.0)$ & $2(0.3)$ & $2(0.2)$ & \\
\hline \multirow{2}{*}{$\begin{array}{l}\text { Commercial } \\
\text { insurance }\end{array}$} & Subscribed & $192(91.9)$ & $579(87.3)$ & $771(88.4)$ & \multirow{2}{*}{$\begin{array}{c}3.192 \\
(p=0.074)\end{array}$} \\
\hline & Unsubscribed & $17(8.1)$ & $84(12.7)$ & $101(11.6)$ & \\
\hline
\end{tabular}

\subsection{The Opinion of the Parents on Using TKM and Their Children's TKM Use Reported} by Parents

Table 3 shows whether the children's TKM use reported by parents changed depending on the parents' ideas or experience in using TKM. This analysis revealed that the group with a child having experienced using TKM had a higher rate of having experienced TKM themselves, compared with the group without a child who experienced TKM $(p<0.001)$. Overall, the participants' satisfaction with the TKM did not show a significant difference. 


\begin{abstract}
Also, parents of the children with experience of using TKM showed a higher level of awareness of TKM $(p=0.033)$. The parents pre-conceived ideas regarding TKM did not result in a statistically significant result. The group with a child who experienced using TKM $(n=198,94.7 \%)$ had a higher number of participants who answered that they were willing to use TKM in the future, compared to children who did not experience using TKM $(n=565,85.2 \%)(p=0.003)$. The group with children with experience of using TKM was more willing to recommend TKM to others $(p<0.001)$.
\end{abstract}

Table 3. Perception of the survey respondents with and without TKM experience of children.

\begin{tabular}{|c|c|c|c|c|c|}
\hline & \multirow{2}{*}{ Variables } & \multicolumn{2}{|c|}{$\begin{array}{l}\text { Existence of TKM Experience of } \\
\text { Children Reported by Parents }\end{array}$} & \multirow{2}{*}{$\begin{array}{l}\text { Total } \\
n(\%)\end{array}$} & \multirow{2}{*}{$\chi^{2}(p)$} \\
\hline & & $\begin{array}{c}\text { Yes } \\
n(\%)\end{array}$ & $\begin{array}{c}\text { No } \\
n(\%)\end{array}$ & & \\
\hline \multirow{6}{*}{$\begin{array}{l}\text { Parent's experience } \\
\text { on TKM use }\end{array}$} & Yes & $191(91.4)$ & 477 (71.9) & $668(76.6)$ & \multirow{6}{*}{$\begin{array}{c}35.220 \\
(p<0.001)\end{array}$} \\
\hline & High school graduate or under & $54(25.8)$ & $154(23.2)$ & $208(23.9)$ & \\
\hline & University or higher school graduate & $137(65.6)$ & $323(48.7)$ & $460(52.8)$ & \\
\hline & No & $18(8.6)$ & $186(28.1)$ & $204(23.4)$ & \\
\hline & High school graduate or under & $9(4.3)$ & $68(10.3)$ & $77(8.8)$ & \\
\hline & University or higher school graduate & $9(4.3)$ & $118(17.8)$ & $127(14.6)$ & \\
\hline \multirow{9}{*}{$\begin{array}{l}\text { Parent's satisfaction } \\
\text { on TKM treatment }\end{array}$} & Satisfied & $143(74.9)$ & $369(77.4)$ & $512(76.6)$ & \multirow{9}{*}{$\begin{array}{c}2.925 \\
(p=0.712)\end{array}$} \\
\hline & High school graduate or under & $42(22.0)$ & $119(24.9)$ & $161(24.1)$ & \\
\hline & University or higher school graduate & $101(52.9)$ & $250(52.4)$ & $351(52.5)$ & \\
\hline & Average & $47(24.6)$ & $102(21.4)$ & $149(22.3)$ & \\
\hline & High school graduate or under & $12(6.3)$ & $34(7.1)$ & $46(6.9)$ & \\
\hline & University or higher school graduate & $35(18.3)$ & $68(14.3)$ & $103(15.4)$ & \\
\hline & Unsatisfied & $1(0.5)$ & $6(1.3)$ & $7(1.0)$ & \\
\hline & High school graduate or under & $0(0.0)$ & $1(0.2)$ & $1(0.1)$ & \\
\hline & University or higher school graduate & $1(0.5)$ & $5(1.0)$ & $6(0.9)$ & \\
\hline \multirow{9}{*}{$\begin{array}{c}\text { Parent's perception } \\
\text { on TKM }\end{array}$} & Well aware & $92(44.0)$ & $234(35.3)$ & $326(37.4)$ & \multirow{9}{*}{$\begin{array}{c}12.125 \\
(p=0.033)\end{array}$} \\
\hline & High school graduate or under & $31(14.8)$ & $71(10.7)$ & $102(11.7)$ & \\
\hline & University or higher school graduate & $61(29.2)$ & $163(24.6)$ & $224(25.7)$ & \\
\hline & Average & $72(34.4)$ & $214(32.3)$ & $286(32.8)$ & \\
\hline & High school graduate or under & $19(9.1)$ & $68(10.3)$ & $87(10.0)$ & \\
\hline & University or higher school graduate & $53(25.4)$ & $146(22.0)$ & $199(22.8)$ & \\
\hline & Not sure & $45(21.5)$ & $215(32.4)$ & $260(29.8)$ & \\
\hline & High school graduate or under & $13(6.2)$ & $83(12.5)$ & $96(11.0)$ & \\
\hline & University or higher school graduate & $32(15.3)$ & $132(19.9)$ & $164(18.8)$ & \\
\hline \multirow{9}{*}{ TKM treatment cost } & Expensive & $135(64.6)$ & $386(58.2)$ & $521(59.7)$ & \multirow{9}{*}{$\begin{array}{c}6.055 \\
(p=0.301)\end{array}$} \\
\hline & High school graduate or under & $46(22.0)$ & $129(19.5)$ & $175(20.1)$ & \\
\hline & University or higher school graduate & $89(42.6)$ & $257(38.8)$ & $346(39.7)$ & \\
\hline & Average & $62(29.7)$ & $239(36.0)$ & $301(34.5)$ & \\
\hline & High school graduate or under & $14(6.7)$ & $77(11.6)$ & $91(10.4)$ & \\
\hline & University or higher school graduate & $48(23.0)$ & $162(24.4)$ & $210(24.1)$ & \\
\hline & Inexpensive & $12(5.7)$ & $38(5.7)$ & $50(5.7)$ & \\
\hline & High school graduate or under & $3(1.4)$ & $16(2.4)$ & $19(2.2)$ & \\
\hline & University or higher school graduate & $9(4.3)$ & $22(3.3)$ & $31(3.6)$ & \\
\hline \multirow{6}{*}{ Intention of re-visit } & Yes & $198(94.7)$ & $565(85.2)$ & $763(87.5)$ & \multirow{6}{*}{$\begin{array}{c}14.195 \\
(p=0.003)\end{array}$} \\
\hline & High school graduate or under & $61(29.2)$ & $189(28.5)$ & $250(28.7)$ & \\
\hline & University or higher school graduate & $137(65.6)$ & $376(56.7)$ & $513(58.8)$ & \\
\hline & No & $11(5.3)$ & $98(14.8)$ & $109(12.5)$ & \\
\hline & High school graduate or under & $2(1.0)$ & $33(3.8)$ & $35(4.0)$ & \\
\hline & University or higher school graduate & $9(4.3)$ & $65(9.8)$ & $74(8.5)$ & \\
\hline \multirow{6}{*}{$\begin{array}{l}\text { Intention of } \\
\text { recommendation }\end{array}$} & Yes & $178(85.2)$ & $427(64.4)$ & $605(69.4)$ & \multirow{6}{*}{$\begin{array}{c}33.184 \\
(p<0.001)\end{array}$} \\
\hline & High school graduate or under & $55(26.3)$ & $145(21.9)$ & $200(22.9)$ & \\
\hline & University or higher school graduate & $123(58.9)$ & $282(42.5)$ & $405(46.4)$ & \\
\hline & No & $31(14.8)$ & $236(35.6)$ & $267(30.6)$ & \\
\hline & High school graduate or under & $8(3.8)$ & $77(11.6)$ & $85(9.7)$ & \\
\hline & University or higher school graduate & $23(11.0)$ & $159(24.0)$ & $182(20.9)$ & \\
\hline
\end{tabular}


The analysis based on the education levels of the participants showed that, of the 191 participants where all the children and the parents experienced TKM, the proportion of those with higher education was higher than the proportion of the same among the participants where either of or all of the parents of the children had not experienced TKM. A similar trend could be observed in the answers to the question whether they are willing to revisit a TKM clinic or recommend it to others.

\subsection{Perception of the TKM Effectiveness for Diseases and Their Children's TKM Use Reported by Parents}

Table 4 shows the analysis result on the perception of the treatment effectiveness for 16 diseases by the parents based on the children's TKM use reported by parents. The group in which the children experienced TKM in 14 out of the 16 diseases showed a higher level of perception on the treatment effect for each of the diseases. Of these, the group where the children experienced TKM for two diseases showed a perception level on the treatment effect that was higher by $10 \%$ compared to the group in which the children never experienced TKM. The said difference was statistically significant (Common cold rhinitis: $p=0.011$, skin disease: $p=0.002$ ).

Table 4. Perception of the TKM effectiveness of diseases of survey respondents with and without TKM experience of children.

\begin{tabular}{|c|c|c|c|c|c|}
\hline \multirow{2}{*}{ Category } & & \multicolumn{2}{|c|}{$\begin{array}{l}\text { Existence of TKM Experience of } \\
\text { Children Reported by Parents }\end{array}$} & \multirow{2}{*}{$\begin{array}{l}\text { Total } \\
n(\%)\end{array}$} & \multirow{2}{*}{$x^{2}(p)$} \\
\hline & & $\begin{array}{l}\text { Yes } \\
n(\%)\end{array}$ & $\begin{array}{c}\text { No } \\
n(\%)\end{array}$ & & \\
\hline \multirow{3}{*}{$\begin{array}{c}\text { Disc related disease } \\
\text { (herniation of intervertebral disc, } \\
\text { spinal stenosis) }\end{array}$} & Effective & $157(75.1)$ & $480(72.4)$ & $637(73.1)$ & \multirow{3}{*}{$\begin{array}{c}2.364 \\
(p=0.307)\end{array}$} \\
\hline & Ineffective & $38(18.2)$ & $115(17.3)$ & $153(17.5)$ & \\
\hline & No idea & $14(6.7)$ & $68(10.3)$ & $82(9.4)$ & \\
\hline \multirow{3}{*}{ Osteoarthritis } & Effective & $158(75.6)$ & $474(71.5)$ & $632(72.5)$ & \multirow{3}{*}{$\begin{array}{c}1.754 \\
(p=0.416)\end{array}$} \\
\hline & Ineffective & $36(17.2)$ & $124(18.7)$ & $160(18.3)$ & \\
\hline & No idea & $15(7.2)$ & $65(9.8)$ & $80(9.2)$ & \\
\hline \multirow{3}{*}{$\begin{array}{l}\text { Frozen shoulder } \\
\text { shoulder pain }\end{array}$} & Effective & $180(86.1)$ & $550(83.0)$ & $730(83.7)$ & \multirow{3}{*}{$\begin{array}{c}1.221 \\
(p=0.543)\end{array}$} \\
\hline & Ineffective & $17(8.1)$ & $69(10.4)$ & $86(9.9)$ & \\
\hline & No idea & $12(5.7)$ & $44(6.6)$ & $56(6.4)$ & \\
\hline \multirow{3}{*}{ Back pain } & Effective & $191(91.4)$ & $568(85.7)$ & $759(87.0)$ & \multirow{3}{*}{$\begin{array}{c}5.166 \\
(p=0.076)\end{array}$} \\
\hline & Ineffective & $9(4.3)$ & $58(8.7)$ & $67(7.7)$ & \\
\hline & No idea & $9(4.3)$ & $37(5.6)$ & $46(5.3)$ & \\
\hline \multirow{3}{*}{ Sprain } & Effective & $189(90.4)$ & $559(84.3)$ & $748(85.8)$ & \multirow{3}{*}{$\begin{array}{c}5.170 \\
(p=0.075)\end{array}$} \\
\hline & Ineffective & $11(5.3)$ & $65(9.8)$ & $76(8.7)$ & \\
\hline & No idea & $9(4.3)$ & $39(5.9)$ & $48(5.5)$ & \\
\hline \multirow{3}{*}{ Facial nerve paralysis } & Effective & $152(72.7)$ & $480(72.4)$ & $632(72.5)$ & \multirow{3}{*}{$\begin{array}{c}0.112 \\
(p=0.946)\end{array}$} \\
\hline & Ineffective & $37(17.7)$ & $123(18.6)$ & $160(18.3)$ & \\
\hline & No idea & $20(9.6)$ & $60(9.0)$ & $80(9.2)$ & \\
\hline \multirow{3}{*}{ Stroke } & Effective & $132(63.2)$ & $412(62.1)$ & $544(62.4)$ & \multirow{3}{*}{$\begin{array}{c}0.560 \\
(p=0.756)\end{array}$} \\
\hline & Ineffective & $52(24.9)$ & $180(27.1)$ & $232(26.6)$ & \\
\hline & No idea & $25(12.0)$ & $71(10.7)$ & $96(11.0)$ & \\
\hline \multirow{3}{*}{ Hypertension } & Effective & $58(27.8)$ & $190(28.7)$ & $248(28.4)$ & \multirow{3}{*}{$\begin{array}{c}0.079 \\
(p=0.961)\end{array}$} \\
\hline & Ineffective & $109(52.2)$ & $339(51.1)$ & $448(51.4)$ & \\
\hline & No idea & $42(20.1)$ & $134(20.2)$ & $176(20.2)$ & \\
\hline \multirow{3}{*}{ Diabetes mellitus } & Effective & $55(26.3)$ & $157(23.7)$ & $212(24.3)$ & \multirow{3}{*}{$\begin{array}{c}0.699 \\
(p=0.705)\end{array}$} \\
\hline & Ineffective & $111(53.1)$ & $358(54.0)$ & $469(53.8)$ & \\
\hline & No idea & $43(20.6)$ & $148(22.3)$ & 191 (21.9) & \\
\hline \multirow{3}{*}{ Digestive disease } & Effective & $128(61.2)$ & $361(54.4)$ & $489(56.1)$ & \multirow{3}{*}{$\begin{array}{c}4.261 \\
(p=0.119)\end{array}$} \\
\hline & Ineffective & $59(28.2)$ & $199(30.0)$ & $258(29.6)$ & \\
\hline & No idea & $22(10.5)$ & $103(15.5)$ & $125(14.3)$ & \\
\hline
\end{tabular}


Table 4. Cont.

\begin{tabular}{|c|c|c|c|c|c|}
\hline \multirow{2}{*}{ Category } & & \multicolumn{2}{|c|}{$\begin{array}{l}\text { Existence of TKM Experience of } \\
\text { Children Reported by Parents }\end{array}$} & \multirow{2}{*}{$\begin{array}{l}\text { Total } \\
n(\%)\end{array}$} & \multirow{2}{*}{$\chi^{2}(p)$} \\
\hline & & $\begin{array}{c}\text { Yes } \\
n(\%)\end{array}$ & $\begin{array}{c}\text { No } \\
n(\%)\end{array}$ & & \\
\hline \multirow{3}{*}{ Common cold rhinitis } & Effective & $124(59.3)$ & $315(47.5)$ & $439(50.3)$ & \multirow{3}{*}{$\begin{array}{c}9.073 \\
(p=0.011)\end{array}$} \\
\hline & Ineffective & $63(30.1)$ & $249(37.6)$ & $312(35.8)$ & \\
\hline & No idea & $22(10.5)$ & $99(14.9)$ & $121(13.9)$ & \\
\hline \multirow{3}{*}{ Dementia } & Effective & $52(24.9)$ & $160(24.1)$ & $212(24.3)$ & \multirow{3}{*}{$\begin{array}{c}1.205 \\
(p=0.548)\end{array}$} \\
\hline & Ineffective & $113(54.1)$ & $339(51.1)$ & $452(51.8)$ & \\
\hline & No idea & $44(21.1)$ & $164(24.7)$ & $208(23.9)$ & \\
\hline \multirow{3}{*}{ Cancer related pain } & Effective & $44(21.1)$ & $149(22.5)$ & $193(22.1)$ & \multirow{3}{*}{$\begin{array}{c}1.461 \\
(p=0.482)\end{array}$} \\
\hline & Ineffective & $116(55.5)$ & $337(50.8)$ & $453(51.9)$ & \\
\hline & No idea & $49(23.4)$ & $177(26.7)$ & $226(25.9)$ & \\
\hline \multirow{3}{*}{ Infertility } & Effective & $72(34.4)$ & $217(32.7)$ & $289(33.1)$ & \multirow{3}{*}{$\begin{array}{c}0.241 \\
(p=0.887)\end{array}$} \\
\hline & Ineffective & $92(44.0)$ & $296(44.6)$ & $388(44.5)$ & \\
\hline & No idea & $45(21.5)$ & $150(22.6)$ & $195(22.4)$ & \\
\hline \multirow{3}{*}{ Skin disease (atopic dermatitis) } & Effective & $107(51.2)$ & $250(37.7)$ & $357(40.9)$ & \multirow{3}{*}{$\begin{array}{c}12.043 \\
(p=0.002)\end{array}$} \\
\hline & Ineffective & $68(32.5)$ & $282(42.5)$ & $350(40.1)$ & \\
\hline & No idea & $34(16.3)$ & $131(19.8)$ & $165(18.9)$ & \\
\hline \multirow{3}{*}{ Genitourinary disease } & Effective & $70(33.5)$ & $188(28.4)$ & $258(29.6)$ & \multirow{3}{*}{$\begin{array}{c}2.104 \\
(p=0.349)\end{array}$} \\
\hline & Ineffective & $92(44.0)$ & $321(48.4)$ & $413(47.4)$ & \\
\hline & No idea & $47(22.5)$ & $154(23.2)$ & $201(23.1)$ & \\
\hline
\end{tabular}

\section{Discussion}

In this study, which was based on the assumption that the experience, opinion, or ideas of parents may influence the use of TKM by their children, the participants were divided into groups based on whether their children had experienced using TKM or not; comparisons were made to evaluate whether these groups showed any differences in terms of the characteristics, awareness, or satisfaction of the parents. This analysis was done for the purpose of developing policies and identifying the ideal time for intervention by understanding the differences in satisfaction and awareness between the two groups.

The initial analysis regarding the participant's children showed that, out of the 5000 participants, $17.4 \%(n=872)$ had a child under the age of 19 . Of these, $24 \%(n=209)$ answered that their children experienced the use of TKM. In previous studies, it was reported that $55.3 \%$ of the target population had a child with TKM use experience [26], and in another, $81 \%$ [25]. However, caution should be practiced when interpreting these results as the target populations were from a daycare center within a self-governing district or the outpatients of a TKM clinic who were familiar with TKM pediatric practices. Especially, the value of $81 \%$ provided in Choi [25] was in contrast with the result of this study, where the proportion of the children who experienced TKM was $17.4 \%$. It is believed to be because of the bias in the selection of the participants, who were selected from the patients who used the TKM clinic of the researcher. In this study, certified national statistics were used and thus based on a representative, standard sample of Korea's general public; therefore, it is believed that the result can be generalized. Also, this study supports the findings in some preceding studies $[25,26]$ that TKM clinics are used to treat the respiratory disease and skin disease in children. However, the said study could not cover the analysis on the purpose for the parents to use TKM clinics due to the limitations of the questions in the questionnaire, making it necessary to use caution in interpreting these findings, as it was assumed using the perception of treatment effect by parents based on the children's TKM use reported by parents.

The reason why the children in Chungcheong and Gyeongsang regions are more likely to use TKM compared to those in the Capital area is attributed to the shortage 
of mainstream medical institutions (e.g., CM institutions and TKM institutions) and the demographics of these areas. These two regions are some of the most representative examples of a combination of urban and rural areas in the same region [27]. Also, the demographics of these areas are heavily leaning toward the older population compared to children [27].

The results of the analysis were used to observe whether there was a difference in the characteristics of the parents depending on the experience of their children with TKM showed that $8.1 \%$ more females (mothers) answered that their children had experienced using TKM. Also, as the participants grew older, being in their $50 \mathrm{~s}$ or older, the rate of answering that their children had experienced using TKM, tended to be higher. This supports the existing study result, where females were more likely to use TKM, and 61.8\% of the users of TKM were at least 45 or older [28]. However, this study does not clarify the factors and correlations. Therefore, care is needed as one attempts to interpret it. Future studies are needed to investigate what is the true tendency of female and people in their $50 \mathrm{~s}$ or older in their use of TKM and what are the factors that contribute to such a result. Based on such findings, it would be possible to employ a more detailed approach to the use of TKM by the children.

On the other hand, the fact that those in their $30 \mathrm{~s}$ or $40 \mathrm{~s}$ were less likely to use TKM, compared to those in their $50 \mathrm{~s}$ or older, can be attributed to the fact that the younger group corresponds with the prime age of workers, namely those aged 25 to 49 [29], a time when individuals are the most active in terms of economic activities. In addition, if both parents of the children are working, it would be more likely that they would experience time or money obstacles, when trying to seek out using TKM. The findings of this study suggest that if the accessibility to TKM is improved for individuals in their $50 \mathrm{~s}$, the younger parents in their $30 \mathrm{~s}$ to $40 \mathrm{~s}$, and females, it would be possible to improve accessibility for their children. Also, in the group where the children had experienced TKM, the proportion of those with a higher level of education was higher among those who gave positive answers concerning the experience of the parents, intent to use in the future, and willingness to recommend. This can be interpreted to be because of the financial resources, desire to be healthy, and interest among these higher-education groups. However, further study is needed in order to clarify this correlation.

About $60 \%$ of the participants answered that TKM treatments were more expensive, which is related to the reimbursement ratio of the health care insurance of Korea. As of 2019 , the reimbursement coverage rate by the health care insurance over the entirety of medical institutions in Korea was $64.2 \%$, while the rate for TKM clinics was 54\% and TKM hospitals was $28.7 \%$ [30]. This is based on the national policy that the health care insurance coverage is to be provided for the treatments with a clear scientific basis to treat severe diseases. While CM treatments have accumulated scientific evidence all over the world, CAM treatments and traditional treatments differ between countries, making it difficult to build up scientific evidence. Therefore, it is necessary to accumulate scientific evidence for the treatment effectiveness and safety by means of exchange and harmonization of traditional medicine and CAM with international organizations such as the World Health Organization taking the leading role. If such a worldwide basis of evidence is built, it could be possible to integrate traditional medicine and CAM treatments into the healthcare coverage, eventually contributing to the improvement of the health of the public.

Children of parents with an experience of using TKM were more likely to use TKM, compared with children whose parents had no experience with using TKM. Of the children whose parents experienced using TKM, 91.4\% used TKM $(n=191)$ themselves, while $71.9 \%$ of the children whose parents never used TKM used it $(n=477)(p<0.001)$. This indicates that the parents' experience with using TKM had a significant impact (20\%) on the use of TKM by their children. The Ministry of Health and Welfare developed the TKM health promotion program for toddlers and infants in 2016 [31], which has gradually been implemented through community health centers [32]. As such, in order for the State to maximize the effectiveness of childhood health management, mainly via TKM, a policy- 
based approach is needed such that the parents of these children may be provided with health management programs through TKM, as well.

In the group of parents whose children experienced using TKM, the rate of answering that they were willing to revisit a TKM clinic was higher by $9.5 \%$, while the rate of answering that they were willing to recommend to others was higher by $20.8 \%(p=0.003)$. This indicates that the perception of the parents impacted the use of TKM by their children, and the improvement of the perception of these parents may have an impact on the use of TKM by their children. It is necessary to conduct further studies in order to clarify these correlations.

The limitations of this study were as follows: first, due to the limitations in the data gathered for this study, it was not possible to clarify the correlations between the parents experience, awareness, and satisfaction with the use of TKM or the use of TKM by their children. Additional studies are needed in order to clarify which variables among the parents' awareness, the purpose of visit, sex, or age, etc., had an impact on the use of TKM by their children. Second, the data obtained through the survey was based on the memories of parents, who were the participants of the survey. Therefore, it is still possible that they answered incorrectly when asked about their children's experience with TKM. Third, the age group of the children of the participants could have had an impact on the experience of using a TKM clinic. However, it was not possible to obtain information on the age of the children. That is, younger children were more likely to have not experienced TKM, which could have contributed to the outcome. Lastly, due to the limitations in the questionnaires, it was not possible to identify the type of treatment the participating patients received. Among the CAM treatments [5,33], vitamins and minerals, probiotics, yoga, qigong, meditation, tai chi, relation techniques, and hypnotherapy are rarely used in TKM clinics, and health insurance coverage of TKM treatment [11] includes acupuncture, electro-acupuncture, pharmacopuncture, herbal medicine, chuna, cupping, and moxibustion. Therefore, it is difficult to compare the usage status and perception of TKM and CAM at the same level and generalize the results of this study.

The strength of this study is that it was conducted using data that is representative of the general Korean population, making the study more generalizable so that the study results can be used as a resource for the government to develop relevant policies. Also, it would be necessary to conduct an in-depth analysis on the decision-makers who decide which medical services to be used, as it is likely that the selection of the medical services used by a child is influenced by parental decisions.

In the future, the following strategies will be needed for the popularization of TKM. First, it is necessary to obtain precise statistical data on the factors and usage of TKM by improving the questionnaire items for the national survey in the future. Based on this study, it was possible to understand that the awareness of the family members on TKM could have an impact on the use of TKM by other members. However, due to the limitations in the question items in the questionnaire, we had difficulties in the analysis of correlations and factors. During the subsequent round of the national survey, the following supplementations are believed to be necessary: (1) Add questions regarding the experience of talking with a family physician or the experience of actually using the TKM for a disease or symptoms; (2) add more question items to ask whether the parents jobs were related to health care or they were actually healthcare professional, and if so, what type of healthcare professionals; (3) add more question items regarding awareness, the intent of use in the future, intent of the recommendation, and an item regarding the preference toward TKM; (4) more survey items for the children, regarding their age, education, and treated interventions and diseases; and (5) if questions are asked about the use of TKM by the children, conduct a face-to-face interview of the parents and the children at the same time. If the above-mentioned items are supplemented, it would be possible to clarify the point of intervention through policies by means of statistical analyses. Second, government-level standardization is required for the Clinical Practice Guideline (CPG) and the Clinical Pathway (CP) centered around the diseases for which TKM has an 
advantage over CM. Choose the diseases for which TKM has an advantage in different stages of the life cycle and develop corresponding CPGs and CPs (e.g., children; atopic dermatitis, females; dysmenorrhea, adults; back pain, seniors; osteoarthritis). With the policies to include these into the coverage of health care, it would have an impact on the family members of TKM users, contributing to popularization. In particular, the use and awareness of TKM by a female parent is likely to have an impact on her child. Therefore, it is necessary to survey the diseases in more details when it comes to female participants (e.g., menstruation, sub-fertility, post-natal management, climacterium, and menopause).

\section{Conclusions}

The present study investigated the correlation between parents' perception and the existence of children's experience with TKM by analyzing the 2017 national survey of TKM usage. The results indicate that the parents' experience of using TKM and their awareness contributed to the differences in their children's experience of using TKM. Our study suggests that the parent's experience of using TKM could have an impact on the children's experience of using TKM. In the future, policy-based interventions would have to be considered for the parents when establishing TKM policies for their children.

Author Contributions: Conceptualization, J.K. and S.-H.S.; methodology, J.K., J.-K.P. and S.-H.S.; software, J.K.; validation, J.K. and J.-Y.P.; formal analysis, J.K.; investigation, J.K.; resources, J.K.; data curation, J.K.; writing—original draft preparation, J.K. and S.-H.S.; writing—review and editing, J.-Y.P., E.-J.L. and S.-H.S.; visualization, J.K.; supervision, S.-H.S.; project administration, S.-H.S.; funding acquisition, S.-H.S. All authors have read and agreed to the published version of the manuscript.

Funding: This work was supported by the Project of Traditional Korean Medicine Community Care Monitoring and Evaluation funded by the Ministry of Health \&Welfare.

Institutional Review Board Statement: Not applicable.

Informed Consent Statement: Not applicable.

Data Availability Statement: The data will be made available upon reasonable request.

Conflicts of Interest: The authors declare that there are no conflicts of interest regarding the publication of this paper.

\section{References}

1. World Health Organization. WHO Traditional Medicine Strategy: 2014-2023; World Health Organization: Geneva, Switzerland, 2013; Available online: https://www.who.int/medicines/publications/traditional/trm_strategy14_23/en/ (accessed on 12 December 2020).

2. Abuduli, M.; Ezat, W.P.; Aljunid, S. Role of traditional and complementary medicine in universal coverage. Malays. J. Public Health Med. 2011, 11, 1-5.

3. Kristoffersen, A.E.; Stub, T.; Broderstad, A.R.; Hansen, A.H. Use of traditional and complementary medicine among Norwegian cancer patients in the seventh survey of the Tromsø study. BMC Complement. Altern. Med. 2019, 19, 341. [CrossRef] [PubMed]

4. National Center for Complementary and Integrative Health. Complementary, Alternative, or Integrative Health: What's in a Name? 2015. Available online: https:/ / www.nccih.nih.gov/health/complementary-alternative-or-integrative-health-whatsin-a-name (accessed on 12 December 2020).

5. Ding, A.; Patel, J.P.; Auyeung, V. Understanding the Traditional Chinese Medicine (TCM) consultation: Why do patients adhere to treatment? Complement. Ther. Clin. Pract. 2020, 39, 101139. [CrossRef]

6. World Health Organization. WHO Global Report on Traditional and Complementary Medicine 2019; World Health Organization: Geneva, Switzerland, 2019; Available online: http://www.who.int/publications/i/item/978924151536 (accessed on 12 December 2020).

7. Huang, C.W.; Tran, D.N.H.; Li, T.F.; Sasaki, Y.; Lee, J.A.; Lee, M.S.; Chen, F.P. The utilization of complementary and alternative medicine in Taiwan: An internet survey using an adapted version of the international questionnaire (I-CAM-Q). J. Chin. Med Assoc. 2019, 82, 665-671. [CrossRef] [PubMed]

8. Na-Bangchang, K.; Karbwang, J. Traditional herbal medicine for the control of tropical diseases. Trop. Med. Health 2014, 42, 3-13. [CrossRef] 
9. Ko, C.R.; Ku, N.P.; Seol, S.S. A Comparative Study on the Traditional Medicine Policies between Korea and China: Focused on the Second Korean Medicine Development Plan and the 12.5 Traditional Chinese Medicine Development Plan. J. Korea Technol. Innov. Soc. 2014, 17, 421-447.

10. Park, H.L.; Lee, H.S.; Shin, B.C.; Liu, J.P.; Shang, Q.; Yamashita, H.; Lim, B. Traditional medicine in China, Korea, and Japan: A brief introduction and comparison. Evid.-Based Complement. Altern. Med. 2012, 2012, 429103. [CrossRef] [PubMed]

11. Kim, H.T.; Hwang, E.H.; Heo, I.; Cho, J.H.; Kim, K.W.; Ha, I.H.; Shin, B.C. Clinical practice guidelines for the use of traditional Korean medicine in the treatment of patients with traffic-related injuries: An evidence-based approach. Eur. J. Integr. Med. 2018, 18, 34-41. [CrossRef]

12. Suh, N.K.; Kang, T.W.; Heo, S.I.; Lee, H.J.; Kim, D.S.; Lim, B.M.; Jang, S.R.; Hong, K.M.; Jung, S.H.; Oh, Y.H. The Korean Health System Based on Healthcare Indicators; Korea Institute for Health and Social Affairs: Sejong, Korea, 2016.

13. Ministry of Health and Welfare; National Development Institute of Korean Medicine; Gallup Korea. 2017 Years National Survey for Traditional Korean Medicine (TKM) Usage; National Development Institute of Korean Medicine: Seoul, Korea, 2018; Available online: https:/ / www.koms.or.kr/board/researchReport/view.do?post_no=45\&menu_no=21 (accessed on 17 December 2020).

14. Kim, J.W.; Choi, J.S. An analysis of family structure on children's medical utilization. Korean J. Soc. Welf. 2016, 68, 5-27.

15. Blackwell, D.L.; Hayward, M.D.; Crimmins, E.M. Does childhood health affect chronic morbidity in later life? Soc. Sci. Med. 2001, 52, 1269-1284. [CrossRef]

16. Larsson, I.; Svedberg, P.; Arvidsson, S.; Nygren, J.M.; Carlsson, M. Parents' experiences of an e-health intervention implemented in pediatric healthcare: A qualitative study. BMC Health Serv. Res. 2019, 19, 800. [CrossRef]

17. An, J.S.; Kim, H.J. A study on the determinants of children and adolescents' health Inequality in Korea. Stud. Korean Youth 2013, 24, 205-231.

18. Lee, Y.W. Family Income and child health gradient in Korea. Health Soc. Welf. Rev. 2014, 34, 7-32.

19. Case, A.; Lubotsky, D.; Paxson, C. Economic status and health in childhood: The origins of the gradient. Am. Econ. Rev. 2002, 92, 1308-1334. [CrossRef]

20. Currie, J. Healthy, wealthy, and wise: Socioeconomic status, poor health in childhood, and human capital development. J. Econ. Lit. 2009, 47, 87-122. [CrossRef]

21. Basu, A.M.; Stephenson, R. Low levels of maternal education and the proximate determinants of childhood mortality: A little learning is not a dangerous thing. Soc. Sci. Med. 2005, 60, 2011-2023. [CrossRef]

22. Braveman, P.; Gottlieb, L. The social determinants of health: It's time to consider the causes of the causes. Public Health Rep. 2014, 129, 19-31. [CrossRef]

23. Loh, C.H. Use of traditional chinese medicine in singapore children: Perceptions of parents and paediatricians. Singap. Med. J. 2009, 50, 1162-1168.

24. Yeh, Y.H.; Chou, Y.J.; Huang, N.; Pu, C.; Chou, P. The trends of utilization in traditional chinese medicine in taiwan from 2000 to 2010: A population-based study. Medicine 2016, 95, e4115. [CrossRef]

25. Choi, Y.J.; Kim, J.H. Survey on outpatients' perception and use of pediatric herbal medicine. J. Korea Inst. Orient. Med. Inform. 1995, 1, 1-24.

26. Park, Y.J.; Lee, S.J.; Yoon, J.Y.; Myoung, S.M. A survey on parent's recognition and utilization patterns of oriental medical care of preschool students in Seong-Dong district. J. Pediatr. Korean Med. 2011, 25, 90-110. [CrossRef]

27. Kim, E.S.; Kim, J.; Kim, B.; Park, K. Classification of regional age structure based on dynamic age structure model and an analysis of association among regional population, economy, education and welfare environment. Korea J. Popul. Stud. 2019, $42,83-113$.

28. Kim, D.S. Study on Factors Related to the Use of Oriental Medical Service among Outpatient. Ph.D. Thesis, Yonsei University, Seoul, Korea, 2013.

29. Choi, Y.S.; Kim, M.Y.; Lim, U. The effects of local labor market characteristics on migration of prime-age workers in Korea. J. Korea Plan. Assoc. 2015, 50, 25-42. [CrossRef]

30. National Health Insurance Service. 2019 Years Survey on the Benefit Coverage Rate of National Health Insurance; National Health Insurance Service: Wonju, Korea, 2020.

31. Park, H.M.; Lee, S.D.; Sung, H.K.; Min, D.L.; Park, S.J.; Sung, D.M. Development of Traditional Korean Medicine Health Promotion Program for Infants and Children; Ministry of Health and Welfare: Sejong, Korea, 2016.

32. Korea Health Promotion Institute. 2021 Integrated Community Health Promotion Program: Traditional Korean Medicine (TKM) Health Promotion. 2020. Available online: https:/ / www.khealth.or.kr/kps/publish/view?menuId=MENU00890\&page_no=B2 017003\&board_idx=10454 (accessed on 13 January 2021).

33. Rodrigues, J.M.; Mestre, M.; Fredes, L.I. Qigong in the treatment of children with autism spectrum disorder: A systematic review. J. Integr. Med. 2019, 17, 250-260. [CrossRef] [PubMed] 